\title{
Análise Crítica da Pesquisa Narrativa
}

\author{
Critical Analysis of Narrative Research \\ Análisis Crítico de la Investigación Narrativa
}

Recebido: 30/06/2021 | Revisado: 06/07/2021 | Aceito: 07/07/2021 | Publicado: 09/07/2021

Tássio Mascarenhas de Carvalho ORCID: https://orcid.org/0000-0001-7416-919X Centro Universitário Campo Limpo Paulista, Brasil Faculdade Estácio de Teresina, Brasil E-mail: metassiocarvalho@gmail.com

Cristiane Rodrigues da Silva

ORCID: https://orcid.org/0000-0001-8178-2048 Centro Universitário Campo Limpo Paulista, Brasil Universidade Federal de São Paulo, Brasil E-mail: cristianeoficiala@gmail.com

Eliane Maria Pires Giavina Bianchi

ORCID: https://orcid.org/0000-0002-2622-2459 Universidade de São Paulo, Brasil Centro Universitário Campo Limpo Paulista, Brasil E-mail: eliane.pires.bianchi@terra.com.br

\section{Resumo}

O contínuo desenvolvimento de abordagens teóricas e metodológicas permitem novas ideias sobre a realidade empírica. A abordagem narrativa permite captar os atores relevantes, suas múltiplas motivações, interesses, atividades e uma interação entre os vários elementos contextuais, fornecendo, assim, um elemento essencial para a compreensão dos vários tipos de processos. É necessária uma base de evidência sobre como os métodos de narrativa podem agregar valor para as formas mais convencionais de pesquisa. A presente pesquisa identifica-se como estratégia associada à abordagem qualitativa, pois estuda-se sobre a pesquisa narrativa a partir de uma análise crítica do levantamento bibliográfico realizado em livros e revistas internacionais. Assim, procurou-se demonstrar a profundidade e o alcance da contribuição da pesquisa narrativa. Esta pesquisa aborda como tema-chave o que caracteriza o desenvolvimento da pesquisa narrativa, perfazendo uma conceituação histórica e tratando sobre a abordagem da pesquisa narrativa, os tipos de coletas de dados e uma análise crítica da pesquisa narrativa.

Palavras-chave: Pesquisa narrativa; Abordagem narrativa; Análise crítica da pesquisa narrativa.

\begin{abstract}
The continuous development of theoretical and methodological approaches allows new ideas about the empirical reality. The narrative approach allows capturing the relevant actors, their multiple motivations, interests, activities and an interaction between the various contextual elements, thus providing an essential element for understanding the various types of processes. A basis for evidence is needed about how narrative methods can add value to more conventional forms of inquiry. The present research identifies itself as a strategy associated to the qualitative approach, since it is studied about the narrative research from a critical analysis of the bibliographical survey carried out in international books and journals. Thus, we sought to demonstrate the depth and scope of the contribution of narrative research. This research addresses as a key theme what characterizes the development of narrative research, making a historical conceptualization and dealing with the approach of narrative research, types of data collection and a critical analysis of narrative research.
\end{abstract}

Keywords:. Narrative research; Narrative approach; Critical analysis of narrative research.

\section{Resumen}

El continuo desarrollo de enfoques teóricos y metodológicos permite nuevas ideas sobre la realidad empírica. El enfoque narrativo permite capturar a los actores relevantes, sus múltiples motivaciones, intereses, actividades y una interacción entre los diversos elementos contextuales, proporcionando así un elemento esencial para la comprensión de los distintos tipos de procesos. Se necesita una base de evidencia sobre cómo los métodos narrativos pueden agregar valor a formas más convencionales de investigación. Esta investigación se identifica como una estrategia asociada al enfoque cualitativo, ya que estudia la investigación narrativa a partir de un análisis crítico de la encuesta bibliográfica realizada en libros y revistas internacionales. Por lo tanto, buscamos demostrar la profundidad y el alcance de la contribución de la investigación narrativa. Esta investigación aborda como tema clave lo que caracteriza el desarrollo de la investigación narrativa, haciendo una conceptualización histórica y abordando el enfoque de la investigación narrativa, los tipos de recolección de datos y un análisis crítico de la investigación narrativa. 
Palabras clave: Investigación narrativa; Enfoque narrativo; Análisis crítico de la investigación narrativa.

\section{Introdução}

A narrativa tem sido utilizada para tratar as experiências, as histórias e as interpretações de acontecimentos (Polkinghorne, 1988). A teoria e o estudo sistemático da narrativa começaram no início do século XX como uma ciência de forma literária. Durante as últimas três décadas, a pesquisa narrativa sofreu uma transformação das ferramentas para análise literária em uma visão mais abrangente e complexa da teoria aplicável às narrativas nunca antes encontradas, por exemplo, estudos no âmbito da narrativa tem se expandido em história, sociologia e antropologia (Riessman, 2002). Em psicologia, uma série de estudos têm abordado a centralidade de narrativas com representações e construções da identidade individual (Bruner, 2002). Colocando em prática a teoria, os terapeutas narrativos visam ajudar os clientes a transformar narrativas negativas de seus problemas em narrativas apontando para possibilidades alternativas de interpretação e ação (White, 2007).

Os primeiros usos explícitos de abordagens narrativas como metodologia de pesquisa na teoria de gestão e organização data da década de 1970 (Clark, 1972; Mitroff; Kilmann, 1978). A maioria desses estudos tomaram como base posição de histórias, mitos, sagas e outras formas de narrativa que eram esquecidas, mas valiosas fontes de dados para pesquisas em organizações. Os estudos com narrativa têm encontrado seu caminho em disciplinas relacionadas com negócios. Dentro dos estudos organizacionais, em especial, a perspectiva narrativa ganhou um terreno sólido ao longo dos últimos 20 anos (Boje, 2001; Czarniawska, 1998; Gabriel, 2000). Também tem sido aplicada a comercialização (Schau; Muniz, 2006) e estudos de gestão (Watson, 2009). No domínio da gestão cultural, pesquisadores têm defendido o uso da narrativa como metodologia de uma abordagem complementar (Soin; Scheytt, 2006).

A pesquisa narrativa como foco de pesquisa sobre a cultura organizacional e simbolismo cresceu na década de $1980 \mathrm{e}$ de 1990, o mesmo aconteceu com o uso de narrativas para explorar o significado da experiência organizacional. Muitas vezes, localizada dentro de um construtivismo social (Boyce, 1996), o uso de narrativas como dados permitiram que pesquisadores examinassem e vivessem simbolicamente dentro das organizações (Gabriel, 1998; Van Buskirk; Mcgrath, 1992).

\section{Metodologia}

Tendo em vista o aspecto social da pesquisa, o presente estudo torna-se relevante porque analisa de forma crítica a pesquisa narrativa. Sem dúvida o tema é atual e desperta interesse, pois o objeto dessa pesquisa identifica a percepção de diversos autores que tratam sobre a pesquisa narrativa. Diante do exposto, assinala-se que a presente pesquisa não pretende esgotar o tema em estudo, devido a sua complexidade e amplitude. Mas, por meio do levantamento bibliográfico, esta pesquisa se apresenta como uma base para novos trabalhos acadêmicos, assim como uma fonte para aqueles que se interessarem pelo tema. Portanto, o presente estudo justifica-se pela possibilidade de abordar as especificidades da pesquisa narrativa, contribuindo, dessa forma, para o desenvolvimento de conhecimentos em torno da pesquisa narrativa e inserindo um olhar crítico sobre a temática.

Hoje, a pesquisa narrativa é reconhecida não apenas como uma forma de dados (Mitroff; Kilmann, 1976), mas também como uma lente teórica (Pentland, 1999), uma abordagem metodológica (Boje, 2001) e várias combinações destes. Ressalta-se que algumas pesquisas tendem a usar o termo "história" em vez de "narrativa", para tratar histórias organizacionais como artefatos in vivo, e enfatizar que sua importância derivou a partir dos fatos que eles forneceram em outros aspectos da organização.

A presente pesquisa, segundo Creswell (2007), identifica-se como estratégia associada à abordagem qualitativa, pois estuda-se sobre a pesquisa narrativa a partir de uma análise crítica do levantamento bibliográfico realizado em livros e revistas 
internacionais. Assim, procura-se demonstrar a profundidade e o alcance da contribuição da narrativa. Esta pesquisa aborda como tema-chave o que caracteriza o desenvolvimento da pesquisa narrativa, perfazendo uma conceituação histórica e tratando sobre a abordagem da pesquisa narrativa, os tipos de coletas de dados, uma análise crítica da pesquisa narrativa e, por fim, as considerações finais.

\section{Referencial Teórico}

Este capítulo está dividido em quatro seções e foi construído de acordo com os fundamentos teóricos encontrados na literatura. A primeira seção apresenta uma breve evolução do conceito de pesquisa narrativa. Em seguida, a segunda seção expõe sobre a abordagem narrativa e a terceira seção aborda sobre os tipos de coletas de dados na pesquisa narrativa. Por fim, a última seção apresenta análise crítica da pesquisa narrativa.

\subsection{Conceito de Pesquisa Narrativa}

O conceito de pesquisa narrativa passou por uma longa evolução no decorrer da história. Apesar de muitos pesquisadores terem definido o termo "pesquisa narrativa", eles não entraram em consenso sobre essa definição. Nesse sentido, será apresentada, em ordem cronológica, a evolução do termo com o intuito de contribuir para uma melhor compreensão desta pesquisa. Antes de iniciar essa apresentação, destaca-se o Quadro 1, que trata de alguns teóricos e os conceitos de pesquisa narrativa empregados em trabalhos publicados em revistas científicas.

Quadro 1. Análise dos conceitos de pesquisa narrativa.

\begin{tabular}{|c|l|}
\hline AUTOR & \multicolumn{1}{|c|}{ CONCEITO DE PESQUISA NARRATIVA } \\
\hline Polkinghorne (1995) & $\begin{array}{l}\text { A pesquisa narrativa tem um foco específico nas histórias contadas } \\
\text { por indivíduos. }\end{array}$ \\
\hline Greenhalgh e Hurwitz (1999) & $\begin{array}{l}\text { A pesquisa narrativa é um conto com um início, uma sequência do } \\
\text { desenrolar dos acontecimentos e um final. }\end{array}$ \\
\hline Riessman (2002) & $\begin{array}{l}\text { As pesquisas narrativas são contos interpretativos que são } \\
\text { reinterpretados por narrativa analista. }\end{array}$ \\
\hline Czarniawska (2004) & $\begin{array}{l}\text { A pesquisa narrativa é entendida como um texto falado ou escrito, } \\
\text { dando conta de um evento/ação ou série de eventos/ações, conectados } \\
\text { cronologicamente. }\end{array}$ \\
\hline Chase (2005) & $\begin{array}{l}\text { A pesquisa narrativa pode ser o termo atribuído a qualquer texto ou } \\
\text { discurso, ou, pode ser texto usado no contexto de um modo de } \\
\text { investigação em pesquisa qualitativa. }\end{array}$ \\
\hline Pinnegar e Daynes (2006) & $\begin{array}{l}\text { A pesquisa narrativa pode ser tanto um método quanto um fenômeno } \\
\text { de estudo. Como método, começa com as experiências expressas em } \\
\text { histórias vividas e contadas de indivíduos. }\end{array}$ \\
\hline
\end{tabular}

Fonte: Autores.

Observa-se, no Quadro 1, que muitos pesquisadores com o intuito de melhor sustentar a sua fundamentação teórica sobre a definição de pesquisa narrativa, ao longo dos anos, foram aperfeiçoando esse conceito. A partir da percepção dos autores, adota-se para a presente pesquisa o conceito apresentado por Czarniawska (2004), pois entende-se que a pesquisa narrativa é a variação de algumas práticas analíticas, texto ou discurso que formam histórias, em ordem cronológica, podendo ser experiências vividas, reportadas ou histórias de indivíduos.

Para Daiute e Lightfoot (2004), a pesquisa narrativa tem muitas formas, usa uma variedade de práticas analíticas e está enraizada em diferentes disciplinas sociais e humanas. Os procedimentos para a implementação desta pesquisa consistem em se concentrar em estudar um ou dois indivíduos, reunir dados através da coleta de suas histórias, relatar experiências 
individuais e ordenar cronologicamente o significado dessas experiências. Embora a pesquisa narrativa tenha se originado da literatura, história, antropologia, sociologia, sociolinguística e educação, diferentes campos de estudo adotaram suas próprias abordagens (Chase, 2005). Em Czarniawska (2004), é encontrada uma orientação organizacional pós-moderna; em Daiute e Lightfoot (2004), uma perspectiva de desenvolvimento humano; uma abordagem psicológica em Lieblich, Tuval-Mashiach e Zilber (1998); em Cortazzi (1993) e Riessman (1993), abordagens sociológicas. Para Chase (2005), estudos sobre pesquisa narrativa é um campo em formação.

As pesquisas narrativas são subjetivas em que elas transmitem a uma pessoa, ou, às vezes, um coletivo, versão de eventos usando uma determinada escolha de palavras, metáforas e estilos. Assim, mesmo quando baseada em acontecimentos reais, nenhuma pesquisa narrativa é uma versão objetiva da verdade. Além disso, as narrativas são intersubjetivas, ou seja, ligam e respondem as subjetividades de seu leitor e ouvinte e são incorporadas em instituições e práticas sociais. A história de uma pessoa, falada em momentos diferentes e por pesquisadores diferentes, nunca é a mesma. Diferentes pessoas contam narrativas diferentes sobre o mesmo evento.

Por todas estas razões, as narrativas devem ser manuseadas com cuidado quando coletadas e usadas como dados de pesquisa. No entanto, utilizadas de forma apropriada, as pesquisas narrativas podem complementar as formas mais convencionais de investigação.

Uma narrativa é semelhante a um processo em que se examinam eventos em um contexto particular em uma estrutura temporal (Elliott, 2005; Langley, 1999; Pentland, 1999). A forma narrativa é particularmente poderosa na construção de um entendimento contextual, também uma virtude em processo de investigação, de como as atividades e os eventos acontecem em circunstâncias específicas. Além disso, os dados de entrevistas e registros históricos muitas vezes possuem qualidades da narrativa (Kjellberg; Andersson, 2003; Pentland, 1999), uma vez que incluem narrativas sobre o que aconteceu, quem fez e quando (Langley, 1999).

Uma narrativa é gerada por um narrador que cria a narrativa de experiência, escolhendo os elementos relevantes e integrando-os em uma entidade (Coffey; Atkinson, 1996; Czarniawska, 2004; Elliott, 2005), é uma história com uma estrutura, consistindo de um início, meio e fim. A trama se conecta a eventos, atividades, atores e contexto, sendo os elementos que compõem o processo de qualquer fenômeno (Santos, 1992). Para Juho, Mainela e Pernu (2010), a abordagem narrativa é inerentemente no mesmo comprimento de onda, como a rede de pesquisa no processo de conceituações que constroem sobre a evolução das relações e eventos que resultam da interação humana.

Uma narrativa é uma contação com um início, uma sequência do desenrolar dos acontecimentos e um final (Greenhalgh, 1999). Ela coloca personagens, eventos, ação no contexto de um conjunto de forma a fazer sentido, e, de modo geral, segue uma forma reconhecível e um padrão (por exemplo, romance, mito ou caso clínico).

\subsection{A abordagem narrativa}

Uma narrativa é uma entidade construída em torno de um enredo em que os elementos narrativos - atividades, eventos, atores, motivos e contexto - estão ligados casualmente uns com os outros (Kjellberg; Andersson, 2003; Elliott, 2005; Polkinchorne, 1995). É uma cadeia de eventos interligados, formando uma estrutura histórica temporal proveniente de um começo e de um fim, que tem sido considerado a principal característica de uma narrativa desde Aristóteles (BOJE, 2001). A temporalidade não necessariamente observa a cronologia, mas ligações de elementos juntos no tempo e indicados com os eventos tem certa ordem (Elliott, 2005).

O narrador produz uma narrativa a partir de sua perspectiva, portanto, refletindo de forma específica orientações subjetivas (Pentland, 1999). O enredo decorre do narrador e a seleção e integração de elementos heterogêneos (atores, 
atividades, eventos, contexto) em uma história (Elliott, 2005). Uma vez que uma única história não pode incluir um número enorme de eventos e atores, o narrador seleciona e classifica o mais importante com base em sua relevância para a história, produzindo causalidade ou caminhos causais para transmitir a mensagem pretendida (Elliott, 2005) na investigação narrativa. O nexo de causalidade significa que uma narrativa fornece um relato de como é um evento relacionado a outra sob um conjunto específico de circunstâncias (Gergen, 1994).

Uma narrativa é construída em torno de um tópico específico e apresentada por uma razão, ou seja, a história tem um ponto que pode variar (Coffey; Atkinson, 1996), ou pode ser bastante inesperada (Elliott, 2005). Uma narrativa pode descrever toda uma história de vida ou organização da história, ou uma descrição de um evento ou processo específico (Flick, 2002).

A narrativa imita o mundo real, reflete e enfatiza aspectos percebidos. Em primeiro lugar, tal como no mundo real, também em uma narrativa, a ação sempre é relacionada ou situada em seu contexto - as configurações físicas (local e tempo) e as configurações sociais (cultura, emoção e cognição) - que, em conjunto, compõem a cena. Em segundo lugar, uma narrativa configura e infunde significados às ações e eventos únicos, integrando-os com outros eventos para produzir processo. Em terceiro lugar, as narrativas são aplicadas a própria compreensão dos ouvintes através de processos cognitivos de percepção e interpretação, ao ponto de que a narração seja esclarecida (Ricoeur, 1984).

A narrativa é um meio que é fácil de construir, compreender, expressar e lembrar (Lende, 2001; Pentaland, 1999), cuja força deriva de cognição narrativa (Bruner, 1986). Elas constroem e transmitem conhecimentos de diferentes percepções: cognição paradigmática que se baseia no conceitual e visa a verdade, considerando que a narrativa se constrói sobre cognição e visa uma abordagem holística da descrição e caminhos causais entre os elementos da narrativa dos atores relevantes, eventos e contextos interligados, levando a uma resolução. Portanto, as narrativas são também meios eficazes de fazer sentido (Bartel; Garud, 2009; Weick, 1969).

As pesquisas narrativas, assim, são variações de algumas práticas analíticas, em que textos ou discursos que formam histórias, em ordem cronológica, podendo ser histórias de indivíduos ou experiências vividas reportadas com foco em um ou poucos indivíduos.

\subsection{Tipos de coletas de dados na pesquisa narrativa}

As pesquisas narrativas podem ser coletadas por meio da entrevista narrativa e entrevista semiestruturada. Na entrevista narrativa, o pesquisador deve ajudar na especificação dos eventos, realizando uma pergunta inicial genérica que deve ser aberta e depois vai especificando com idas e vindas, podendo pedir uma reflexão final do entrevistado. Já a entrevista semiestruturada, o pesquisador faz apenas algumas perguntas predeterminas.

Ressalta-se que as pesquisas narrativas podem ser executadas através de história de vida, história oral (pesquisadores captam reflexões sobre eventos e como antecedentes e consequentes impactam as vidas), estudos biográficos (pesquisadores escrevem a história de uma pessoa), autobiografia ("pesquisador" é o sujeito) ou análise de narrativas, que são conexões de eventos que montam uma história (Polkinghorne, 1995).

Em princípio, o pesquisador entrevista as pessoas que parecem saber mais sobre o tema estudado do que quaisquer outras. Acredita-se que essas pessoas estão no topo de uma hierarquia de credibilidade, isto é, o que dizem é mais verdadeiro do que aquilo que outras, que não conhecem tão bem o assunto, diriam. Na verdade, o pesquisador não deve se limitar a ouvir apenas estas pessoas. Deve também ouvir quem nunca é ouvido, invertendo assim esta hierarquia de credibilidade (Creswell, 2007).

Um dos principais problemas das entrevistas e questionários é detectar o grau de veracidade dos depoimentos. Trabalhando com estes instrumentos de pesquisa é bom lembrar que se lida com o que o indivíduo deseja revelar, o que deseja 
ocultar e a imagem que quer projetar de si mesmo e de outros. A personalidade e as atitudes do pesquisador também interferem no tipo de respostas que ele consegue de seus entrevistados (Denzin, 1989).

As entrevistas e questionários podem ser estruturados de diferentes maneiras, podendo ser rigidamente padronizados, assistemáticos ou entrevista projetiva, que utiliza recursos visuais, como quadros, pinturas, fotos para estimular a resposta dos pesquisados. O pesquisador deve ter em mente que cada questão precisa estar relacionada aos objetivos de seu estudo. As questões devem ser enunciadas de forma clara e objetiva, sem induzir e confundir, tentando abranger diferentes pontos de vista (Clandinin; Connelly, 2000).

Ao construir a entrevista ou o questionário, o pesquisador deve decidir que informação deve ser procurada, o conteúdo da pergunta e como redigir as perguntas, observando o lugar na sequência apresentada. Além disso, o pesquisador deve decidir que tipo de entrevista ou questionário deve ser usado, redigindo um primeiro rascunho e após a crítica de outras pessoas, reexaminar e rever as perguntas, para depois realizar um pré-teste, em que se aplica e discute com os entrevistados as dificuldades. Por fim, o pesquisador reelabora a entrevista ou o questionário (Czarniawska, 2004).

O estudioso precisa estar muito bem preparado antes de abordar o grupo pesquisado, saber o máximo possível e não fazer perguntas desnecessárias, cujas respostas poderiam ser encontradas em outras fontes. Além disso, o pesquisador deve ser o maior conhecedor do tema estudado. Portanto, a entrevista ou questionário são instrumentos para conseguir respostas que o pesquisador não conseguiria com outros instrumentos (Creswell, 2007).

Como qualquer relação pessoal, a arte de uma entrevista bem-sucedida depende fortemente da criação de uma atmosfera amistosa e de confiança. As características pessoais do pesquisador e pesquisado são decisivas. É muito importante não se criar antagonismo ou suspeita nas primeiras abordagens. As atitudes e opiniões do pesquisador não podem aparecer em primeiro plano. Ele deve tentar ser o mais neutro possível, não sugerindo respostas. É sempre útil começar com perguntas mais fáceis e não ir longe demais no início. Destaca-se que o pesquisador precisa respeitar as limitações do pesquisado quanto ao local e ao tempo da entrevista. Também deve-se ir bem preparado para aproveitar ao máximo a entrevista ou o questionário e registrar adequadamente. Por fim, o pesquisador deverá de imediato transcrever as entrevistas a fim de, ao realizar novas entrevistas, não repetir questões e dominar cada vez mais o assunto (Denzin, 1989).

\section{Resultados e Discussão}

\subsection{Análise crítica da pesquisa narrativa}

As narrativas são de fato retóricas, uma narrativa é uma ferramenta de argumentação, destinadas a persuadir o ouvinte da legitimidade da perspectiva do narrador (Greenhalg, 2016).

Relatos narrativos nunca são auto interpretação. Como Eastmond (2007) observou:

A experiência nunca é representada diretamente, mas editada em diferentes fases do processo da vida ao texto. Como o texto publicado, a história é exposta a um público diferente e a outra interpretação. A cultura é assim... central, não apenas na elaboração de uma história de um assunto particular, mas também nos caminhos que os outros compreendam e recontam a história.

Cardel e Soderverg (2010) chamam a atenção dos pesquisadores no sentido de tomar o devido cuidado ao interpretar as narrativas dos entrevistados como fatos objetivos à conversão exata dos acontecimentos, pois esta interpretação poderá ocasionar mal-entendido no status ontológico das histórias que podem consciente ou inconscientemente, terem sido organizadas de forma alterada por este narrador, pois tanto a sua memória bem como a sua percepção sobre a história contada poderá estar com viés cognitivo; ou este poderá utilizar uma estratégia deliberada para editar sua narrativa. Ainda, segundo estes autores, o pesquisador ao entrevistar as pessoas em determinada organização, a narrativa poderá ser apresentada de tal 
maneira cujos objetivos e intenções poderão não estar inteiramente claros.

Gummerson (2005) propõe que as rigorosas técnicas de investigação precisam estar em consonância com as qualidades humanas nas questões que envolvam a intuição, o senso comum, experiência, bom senso, sabedoria, discernimento, conhecimento tácito, empatia e ética. Se o aspecto humano for ignorado, haverá uma investigação irrelevante e errada, poderá ser prejudicial, principalmente quando se trata de pesquisa qualitativa onde o pesquisador continua a ser o instrumento mais importante e em muitas situações, reconhecidamente ou não, a análise e interpretação vai depender também da intuição do indivíduo, mesmo que esta seja considerada como uma anti-ciência.

Primeiro e acima de tudo, narrativas não são verdadeiras em qualquer sentido simples. Denzin (1989), uma das maiores autoridades mundiais em pesquisas qualitativas, definiu como narrativas ficcionais declarações que, num grau variável, são sobre a vida real que se viveu. No entanto, as histórias podem ser pensadas como perspectivas (ou seja, a transmitir uma perspectiva particular) em vez de (ou seja, tendenciosa sistematicamente distorcida), embora às vezes possam ser tanto preconceituosas. Considerando que o termo é utilizado em epidemiologia e têm conotações negativas, o termo investigação narrativo nesta perspectiva tem conotações positivas, uma vez que permite o acesso a uma pessoa de sentimentos e no mundo da vida. No entanto, se as histórias pessoais forem equiparadas com a verdade, isto irá produzir investigação empobrecida.

Gummerson (2005) considera que os pesquisadores devem ser mais críticos em seus dados e suas fontes no sentido de se ter um espírito construtivo com o bom senso e a ética; o rigor e o modo sistemático do investigador.

Uma narrativa é uma versão subjetiva dos eventos. A investigação narrativa inclui a coleta de novas histórias, a ordenação e a reanálise de histórias existentes. As narrativas podem complementar outras formas de prova, mas devem ser coletadas e analisadas com atenção à qualidade. Um conjunto de critérios-chave, para guiar a avaliação crítica de investigação narrativa é proposto; estes critérios incluem medidas para garantir confiabilidade, plausibilidade e criticidade (Greenhalgh, 2016).

Narrativa criteriosa também envolve um trade-off entre o tamanho da amostra e a profundidade de análise. A maioria das entrevistas qualitativas são semiestruturadas (ou seja, o pesquisador passa por uma rápida lista de perguntas). Uma entrevista narrativa (especialmente quando perguntada sobre a história de vida) deve ser feita mais como uma conversa e ser conduzida pela curiosidade do pesquisador em vez de usar um conjunto pré-definido de perguntas prontas (Mishler, 1991; Wengraf, 2001) dessas entrevistas que tendem a ser longas e exigem extensa análise. Correspondentemente, os tamanhos das amostras tendem a ser pequenas (e, portanto, num sentido estatístico, representativas).

Gumerson (2005) entende que é fácil criticar os métodos qualitativos, principalmente quando a crítica estabelece critérios quantitativos a partir de um paradigma. Diante da falta de consenso, Gumerson (2005) reforça que não há necessidade de os pesquisadores qualitativos se preocuparem e se sentirem obrigados a justificar a sua investigação contra uma norma que foi criada pela pesquisa quantitativa; porque a crítica pode ser levantada contra todos os métodos e técnicas, devendo ficar claro ao pesquisador que a direção está no sentido de se ter consciência sobre os pontos fortes e fracos e a relevância para o pesquisador.

Em resumo, as limitações da investigação narrativa são importantes e devem sempre se ter em mente. As narrativas não devem ser totalmente rejeitadas, como uma forma de investigação, mas as narrativas individuais nunca devem ser utilizadas como prova de causalidade direta, ou como uma janela, sem distorções, com as experiências e perspectivas (Greenhalg, 2016). 


\section{Considerações Finais}

Este artigo analisou a conceituação histórica, a abordagem narrativa, os tipos de coletas de dados da pesquisa narrativa e de forma crítica a investigação narrativa. Além disso, o artigo faz uma contribuição teórico-metodológica para a investigação do processo. Foi realizada uma análise e interpretação sobre as diferentes abordagens metodológicas e critérios de qualidade da investigação narrativa; bem como sobre evidências empíricas a partir de uma ampla gama de estudos de pesquisa narrativa.

Mesmo quando baseada em acontecimentos reais, nenhuma narrativa tem como objetivo a versão da verdade (embora importante, ela pode ser dita de um conjunto de números ou respostas da pesquisa). As narrativas são subjetivas no sentido de que uma pessoa (ou, às vezes, um grupo) transmite a versão de eventos utilizando uma determinada escolha de palavras, metáforas e estilos. As narrativas são também intersubjetivas no sentido de ligar e responder as subjetividades de seu (s) leitor e ouvinte (s) e são incorporadas em instituições e práticas sociais. Portanto, a história de uma pessoa, dita duas vezes, nunca é a mesma.

Qualquer que seja a técnica utilizada, os principais critérios de qualidade para a investigação narrativa incluem medidas para garantir confiabilidade, plausibilidade e criticidade. Por todas estas razões, as narrativas devem ser manuseadas com cuidado quando coletadas e utilizadas como dados de pesquisa. No entanto, se utilizadas de forma apropriada, as narrativas podem complementar as formas mais convencionais de investigação.

Se filosoficamente aceitar que existe uma realidade, terá de ajustar os métodos para compreender a realidade da melhor forma possível. O que não se pode fazer é dizer que a realidade não se encaixa com o paradigma dominante e suas técnicas. Toda a realidade é adepta à investigação científica e todo o esforço para se concentrar apenas em um número limitado de pesquisador e questões que impedem o desenvolvimento e a utilidade da investigação.

Apesar do potencial representado na narrativa, uma abordagem cuidadosa deve ser dada às narrativas no sentido de verificar se elas estão verdadeiramente aptas para servirem a fins de investigação, e qual o papel que a abordagem narrativa poderia certamente ser aplicada: se a narrativa de dados pode ser integrada com outros tipos de dados ou se a análise da narrativa pode oferecer novas dimensões teóricas.

Uma maneira de continuar esta pesquisa seria no futuro introduzir mais discursos para o material empírico analisando narrativas, se possível, sobre os mesmos eventos coletados. Portanto, futuras pesquisas poderiam continuar a elaboração da abordagem narrativa e as técnicas da análise narrativa na investigação do processo para que possam atender melhor os diferentes pesquisadores.

\section{Referências}

Bartel, C. A.; \& Garud, R. (2009). The role of narratives in sustaining organizational innovation. Organization Science, 20(1), $107-117$.

Boje, D. M. (2001) Narrative Methods for Organizational and Communication Research. Sage.

Boyce, M. E. (1996) Organizational story and story-telling: a critical review. Journal of Organizational Change Management, 9, 5-26.

Bruner, J. (1986) Actual minds, possible words. Harvard University Press.

Bruner, J. (1990) Acts of meaning. Harvard Univer- sity Press.

Bruner, J. (2002) Making stories. Law, Literature, Life. Harvard University Press.

Chase, S. (2005). Narrative inquiry: Multiple lenses approaches, voices. In N. K. Denzin and Y. S. Lincoln (Eds.), The Sage handbook of qualitative research (3rd ed, pp. 651-680). Sage.

Clandinin, D. J., \& Connelly, F. M. (2000). Narrative inquiry: Experience and story in qualitative research. Jossey-Bass.

Clark, B. R. (1972) The organizational saga in higher education. Administrative Science Quarterly, 17, $178-184$.

Coffey, A., \& Atkinson, P. (1996) Making sense of qualitative data. Complementary research strategies. Sage Publications. 
Research, Society and Development, v. 10, n. 8, e54510817743, 2021

(CC BY 4.0) | ISSN 2525-3409 | DOI: http://dx.doi.org/10.33448/rsd-v10i8.17743

Cortazzi, M. (1993) Narrative analysis. Falmer Press.

Creswell, J. (2007) Projeto de pesquisa: métodos qualitativo, quantitativo e misto. Bookman.

Curtis, R. (1994). Narrative form and normative force: Baconian story-telling in popular science. Social Studies of Science, 24(3), 419-461.

Czarniawska, B. (1998). A Narrative Approach to Organization Studies. Sage.

Czarniawska, B. (2004). Narratives in social science research. Sage.

Daiute, C., \& Lightfoot, C. (2004). Narrative analysis: Studying the development of individuals in society. Sage.

Denzin, N. K. (1989). Interpretive Biography. Sage.

De Waard, A. (2010). From Proteins to Fairytales: Directions in Semantic Publishing, Intelligent Systems, IEEE, 25(2), 83-88.

Eastmond, M. (2007). Stories as lived experience: narratives in forced migration research. J Refug Stud. 20(2):248-64.

Elliiot, J. (2005). Using narrative in social research: Qualitative and quantitative approaches. Sage.

Flick, U. (2002). An introduction to qualitative research. Sage.

Gabriel, Y. (1998). The use of stories. In Symon, G. and Cassell, C. (eds), Qualitative Methods and Analysis in Organizational Research: A Practical Guide. Sage, pp. $135-160$.

Gabriel, Y. (2000). Storytelling in Organizations, Facts, Fictions, and fantasies. Oxford University Press.

Gergen, K. (1994). Realities and relationships: Soundings in social construction. Harvard University Press.

Greenhalgh, T. (2016). Cultural contexts of health: the use of narrative research in the health sector. WHO Regional Office for Europe.

Greenhalgh, T., \& Hurwitz, B. (1999). Why study narrative? BMJ. 318(7175):48-50.

Juho, A., Mainela, T., \& Pernu, E. (2010). Levels of processes, intertwining actions and network change. Paper presented at the. 26th IMP Conference, September 2-4, 2010, Budapest, Hungary.

Kjellberg, H., \& Andersson, P. (2003). Where is the action? The reconstruction of action in business narratives. In A. Woodside (Ed.), Advances in Business Marketing and Purchasing, Evaluating marketing actions and outcomes (pp. 13-58). Elsevier Science.

Labov, W., \& Waletzky, J. (1967). Narrative analysis: Oral versions of personal experience. In J. Helm (Ed.), Essays on the verbal and visual arts (pp. 12-44). Seattle: University of Washington Press.

Langley, A. (1999). Strategies for theorizing from process data. The Academy of Management Review, 24(4), 691-710.

Linde, C. (2001). Narrative and social tacit knowledge. Journal of Knowledge Management, 5(2), 160-170.

Lieblich, A., Tuval-Mashiach, R., \& Zilber, T. (1998). Narrative research: Reading, analysis, and interpretation. Sage.

Mishler, E. G. Research interviewing. Harvard University Press, 1991.

Mitroff, I. I.; Kilmann, R. H. Methodological Approaches to Social Science. Jossey-Bass, 1978.

Mitroff, I. I., \& Kilmann, R. H. (1976). On organizational stories: an approach to the design and analysis of organizations through myths and stories. In Kilmann, R.H., Pondy, L.R. \& Slevin, D.P. The Management of Organizational Design, pp. 189-207.

Parker, M. (2002). Against management: Organisation in the Age of Managerialism. Cam- Polity.

Pentland, B. T. (1999). Building process theory with narrative: from description to explanation. Academy of Management Review, 24, 711-724.

Pinnegar, S., \& Daynes, J. G. (2006). Locating narrative inquiry historically: Thematics in the turn to narrative. In D. J. Clandinin (Ed.), Handbook of narrative inquiry. Sage.

Polkinghorne, D. E. (1995). Narrative configuration in qualitative analysis. Qualitative Studies in Education, 8, 5-23.

Polkinghorne, D. E. (1988). Narrative Knowing and the Human Sciences. State University of New York.

Riessman, C. K. (1993). Narrative analysis. Sage.

Riessman, C. K. (2002). Analysis of personal narratives. In J. F. Gubrium, \& J. A. Holstein (Eds.), Handbook of interview research (pp. 695-709). Thousand Sage Publications.

Ricoeur, P. (1983). Time and Narrative, Vol. 1 (trans. K. McLaughlin and D. Pellauer). University of Chicago Press.

Schau, H. P., \& Mun iz, A. M. (2006). A tale of tales: The Apple Newton narratives. Journal of Strategic Marketing, 14, 19-33. 
Research, Society and Development, v. 10, n. 8, e54510817743, 2021

(CC BY 4.0) | ISSN 2525-3409 | DOI: http://dx.doi.org/10.33448/rsd-v10i8.17743

Sheehan, J., \& Rode, S. (1999). On scientific narrative: Stories of light by Newton and Einstein. Journal of Business and Technical Communication, 13 (3), p. 336-358.

Soin, K., \& Scheytt, T. (2006). Making the case for narrative methods in cross-cultural organizational research. Organizational Research Methods, 9 (1), 5577 .

Van Buskirk, W., \& Mcgrath, D. (1992). Organizational stories as a window on affect in organizations. Journal of Organizational Change Management, 5, 924.

Watson, T. J. (2009). Narrative, life story and manager identity: A case study in autobiographical identity work. Human Relations, 62(3), 425-452.

Weick, K. E. (1969). The social psychology of organizing. Addison-Wesley Publishing Company, Reading.

Wengraf T. (2001). Qualitative research interviewing: biographic narrative and semi- structured methods. Sage.

White, M. (2007). Maps of narrative practice. WW Norton. 